\title{
A NEW PROOF OF \\ THE AHLFORS FIVE ISLANDS THEOREM
}

By

\author{
WALTER BERGWEILER
}

\begin{abstract}
We deduce the Ahlfors five islands theorem from a corresponding result of Nevanlinna concerning perfectly branched values, a rescaling lemma for non-normal families and an existence theorem for quasiconformal mappings. We also give a proof of Nevanlinna's result based on the rescaling lemma and a version of Schwarz's lemma.
\end{abstract}

\section{Results of Ahlfors, Nevanlinna and Zalcman}

Let $D_{j}, j=1, \ldots, 5$, be simply-connected domains on the Riemann sphere $\hat{\mathbb{C}}$ with piecewise analytic boundary and pairwise disjoint closures. Let $D \subset \mathbb{C}$ be a domain and denote by $\mathcal{F}_{A}(D)=\mathcal{F}_{A}\left(D,\left\{D_{j}\right\}_{j=1}^{5}\right)$ the family of all meromorphic functions $f: D \rightarrow \hat{\mathbb{C}}$ with the property that no subdomain of $D$ is mapped conformally onto one of the domains $D_{j}$ by $f$. (If there is such a subdomain, then it is called a simple island over $D_{j}$.) The Ahlfors five islands theorem can now be stated as follows.

Theorem A.1. $\mathcal{F}_{A}\left(D,\left\{D_{j}\right\}_{j=1}^{5}\right)$ is normal.

Closely related is the following result.

Theorem A.2. $\mathcal{F}_{A}\left(\mathbb{C},\left\{D_{j}\right\}_{j=1}^{5}\right)$ contains only the constant functions.

These results come from the Ahlfors theory of covering surfaces; see [5], [22, Chapter 5], [28, Chapter XIII] or [34, Chapter VI].

In order to state two similar results associated with Nevanlinna's theory on the distribution of values, let $a_{j} \in \hat{\mathbb{C}}, j=1, \ldots, 5$, be distinct and denote for a domain $D \subset \mathbb{C}$ by $\mathcal{F}_{N}(D)=\mathcal{F}_{N}\left(D,\left\{a_{j}\right\}_{j=1}^{5}\right)$ the family of all meromorphic functions $f: D \rightarrow \hat{\mathbb{C}}$ with the property that there does not exist $j \in\{1, \ldots, 5\}$ such that $f$ has a simple $a_{j}$-point. (The value $a_{j}$ is then called perfectly branched or totally ramified.) 
Theorem N.1. $\mathcal{F}_{N}\left(D,\left\{a_{j}\right\}_{j=1}^{5}\right)$ is normal.

Theorem N.2. $\mathcal{F}_{N}\left(\mathbb{C},\left\{a_{j}\right\}_{j=1}^{5}\right)$ contains only the constant functions.

Nevanlinna himself $([27$, p. 102], $[28, \S X .3])$ was concerned more with Theorem N.2, while Theorem N.1 appears in the work of Bloch [15, Theorem XLIV] and Valiron [35, Theorem XXVI] (with proofs being based on Nevanlinna's theory). Among further accounts of Nevanlinna theory we mention [22, Chapters 1-4] and [34, Chapter V]; for a proof of Theorems N.1 and N.2 using different methods we refer to Robinson [29].

Theorem N.1 follows from Theorem A.1 and Theorem N.2 follows from Theorem A.2 because if $a_{j} \in D_{j}$ for $j \in\{1, \ldots, 5\}$, then $\mathcal{F}_{N}\left(D,\left\{a_{j}\right\}_{j=1}^{5}\right) \subset$ $\mathcal{F}_{A}\left(D,\left\{D_{j}\right\}_{j=1}^{5}\right)$; and, given five distinct values $a_{j}$, one can always find five domains $D_{j}$ with the required properties such that $a_{j} \in D_{j}$ for $j \in\{1, \ldots, 5\}$ (for example, one can choose $D_{j}$ to be a sufficiently small disk around $a_{j}$ ).

Also, Theorem A.2 follows from Theorem A.1 and Theorem N.2 follows from Theorem N.1 because if $f: \mathbb{C} \rightarrow \hat{\mathbb{C}}$ is non-constant, then $\mathcal{F}=\{f(n z)\}_{n \in \mathbb{N}}$ is not normal at 0 , but $\mathcal{F} \subset \mathcal{F}_{X}(\mathbb{C})$ if $f \in \mathcal{F}_{X}(\mathbb{C})$, for $X \in\{A, N\}$.

It is less obvious (and perhaps less well-known) that in turn Theorem X.1 may also be deduced from Theorem X.2 for $\mathrm{X}=\mathrm{A}$ or $\mathrm{X}=\mathrm{N}$. This follows from the following

Rescaling Lemma. Let $D \subset \mathbb{C}$ be a domain and let $\mathcal{F}$ be a family of functions meromorphic in $D$. If $\mathcal{F}$ is not normal, then there exist a sequence $\left(z_{k}\right)$ in $D, a$ sequence $\left(\rho_{k}\right)$ of positive real numbers, a sequence $\left(f_{k}\right)$ in $\mathcal{F}$, a point $z_{0} \in D$ and a non-constant meromorphic function $f: \mathbb{C} \rightarrow \hat{\mathbb{C}}$ whose spherical derivative is bounded such that $z_{k} \rightarrow z_{0}, \rho_{k} \rightarrow 0$ and $f_{k}\left(z_{k}+\rho_{k} z\right) \rightarrow f(z)$ locally uniformly in $\mathbb{C}$.

This lemma is due to Zalcman [36]. The corresponding result for normal functions had been proved earlier by Lohwater and Pommerenke [25]. The proof of the rescaling lemma is fairly short and elementary; it uses only Marty's criterion. We remark that the statement that $f$ has bounded spherical derivative does not appear in [36] but follows immediately from the proof; see also [37, p. 216f]. For completeness, we include a proof of the rescaling lemma in $\S 4$.

To deduce Theorem X.1 from Theorem X.2, we have only to note that if $\mathcal{F}_{X}(D)$ were not normal, then we could find sequences $\left(z_{k}\right),\left(\rho_{k}\right)$ and $\left(f_{k}\right)$ as in the rescaling lemma and would obtain a non-constant function $f \in \mathcal{F}_{X}(\mathbb{C})$, contradicting Theorem X.2.

In this note we show that the rescaling lemma, together with an existence theorem for quasiconformal mappings, can also be used to deduce Theorem A.2 
from Theorem N.2. We also show that the rescaling lemma and a generalization of Schwarz's Lemma give a proof of Theorems N.1 and N.2. We thus provide an alternative approach to the Ahlfors five islands theorem.

For a generalization of the above results, a discussion of applications of the Ahlfors five islands theorem in complex dynamics, and some further comments, see $\S 5$ below.

\section{Acknowledgments}

I am grateful to W. Cherry and D. Minda for pointing out reference [29] and to A. Eremenko for drawing my attention to [14]. I also thank D. Bargmann, M. Bonk, W. Cherry, A. Eremenko and L. Zalcman for useful discussions and comments.

\section{The proof that Theorem N.2 implies Theorem A.2}

Besides the rescaling lemma, we shall need the following existence theorem for quasiconformal mappings (see [7, Chapter V] or [24, Chapter V]).

Existence Theorem. Let $\mu: \mathbb{C} \rightarrow \mathbb{C}$ be measurable with $\|\mu\|_{\infty}<1$. Then there exists a quasiconformal homeomorphism $\phi: \mathbb{C} \rightarrow \mathbb{C}$ whose complex dilatation agrees with $\mu$ almost everywhere.

This existence theorem is rather deep. It will be apparent from the arguments below, however, that for our purposes it suffices to know that the conclusion holds for smooth $\mu$. In this case the proof is easier. For a discussion of the existence theorem and its history we refer to [23, p. 140].

The following result is a simple consequence of the existence theorem (cf. [24, Chapter VI]).

Corollary. Let $q: \mathbb{C} \rightarrow \hat{\mathbb{C}}$ be quasiregular. Then there exists a quasiconformal homeomorphism $\phi: \mathbb{C} \rightarrow \mathbb{C}$ and a meromorphic function $g: \mathbb{C} \rightarrow \hat{\mathbb{C}}$ such that $q=g \circ \phi$.

To deduce the corollary from the existence theorem, we denote the complex dilatation of $q$ by $\mu$, choose $\phi$ according to the existence theorem, define $g=q \circ \phi^{-1}$ and observe that the complex dilatation of $g$ vanishes almost everywhere, which implies that $g$ is meromorphic.

To deduce Theorem A.2 from Theorem N.2, we assume that Theorem N.2 holds, but that there are domains $D_{j}$ as in $\S 1$ such that $\mathcal{F}_{A}\left(\mathbb{C},\left\{D_{j}\right\}_{j=1}^{5}\right)$ contains 
a non-constant function $f$. We may assume that $\infty$ is not in the closure of the domains $D_{j}$, because otherwise we can replace $f$ by $T \circ f$ and $D_{j}$ by $T\left(D_{j}\right)$ for a suitable Möbius transformation $T$. Fix five distinct values $a_{1}, \ldots, a_{5} \in \mathbb{C}$. For $0<\varepsilon<\frac{1}{2} \min _{j \neq k}\left|a_{j}-a_{k}\right|$, the closures of the disks $D\left(a_{j}, \varepsilon\right)=\left\{z \in \mathbb{C}:\left|z-a_{j}\right|<\varepsilon\right\}$ are pairwise disjoint. It is not difficult to see that there exists a quasiconformal homeomorphism $\psi_{\varepsilon}: \mathbb{C} \rightarrow \mathbb{C}$ such that $\psi_{\varepsilon}\left(D_{j}\right) \subset D\left(a_{j}, \varepsilon\right)$ for all $j \in\{1, \ldots, 5\}$. Now $\psi_{\varepsilon} \circ f$ is quasiregular, and thus the corollary to the existence theorem implies that there exist a quasiconformal homeomorphism $\phi_{\varepsilon}: \mathbb{C} \rightarrow \mathbb{C}$ and a meromorphic function $g_{\varepsilon}: \mathbb{C} \rightarrow \hat{\mathbb{C}}$ such that $\psi_{\varepsilon} \circ f=g_{\varepsilon} \circ \phi_{\varepsilon}$. Since $f \in \mathcal{F}_{A}\left(\mathbb{C},\left\{D_{j}\right\}_{j=1}^{5}\right)$ and $\psi_{\varepsilon}\left(D_{j}\right) \subset D\left(a_{j}, \varepsilon\right)$ for $j \in\{1, \ldots, 5\}$, we have $g_{\varepsilon} \in \mathcal{F}_{A}\left(\mathbb{C},\left\{D\left(a_{j}, \varepsilon\right)\right\}_{j=1}^{5}\right)$.

Fix a sequence $\left(\varepsilon_{k}\right)$ tending to zero. We may assume that $\left\{g_{\varepsilon_{k}}\right\}$ is not normal, because otherwise we can replace $g_{\varepsilon_{k}}(z)$ by $g_{\varepsilon_{k}}\left(M_{k} z\right)$ with $M_{k}$ large. Now apply the rescaling lemma to the family $\left\{g_{\varepsilon_{k}}\right\}$ to obtain a subsequence of $\left(g_{\varepsilon_{k}}\right)$, which we again denote by $\left(g_{\varepsilon_{k}}\right)$, and sequences $\left(z_{k}\right)$ and $\left(\rho_{k}\right)$ as in the rescaling lemma, such that $g_{\varepsilon_{k}}\left(z_{k}+\rho_{k} z\right) \rightarrow g(z)$ locally uniformly in $\mathbb{C}$ for a non-constant meromorphic function $g$. Since $\varepsilon_{k} \rightarrow 0$, we have $g \in \bigcap_{\varepsilon>0} \mathcal{F}_{A}\left(\mathbb{C},\left\{D\left(a_{j}, \varepsilon\right)\right\}_{j=1}^{5}\right)=$ $\mathcal{F}_{N}\left(\mathbb{C},\left\{a_{j}\right\}_{j=1}^{5}\right)$. This contradicts Theorem N.2.

\section{A proof of Theorems N.1 and N.2}

Besides the rescaling lemma, we shall require an extension of Schwarz's Lemma. The natural generalization of Schwarz's Lemma to multivalued functions is due to Nehari [26]. For our purposes, a special case of Nehari's result suffices.

Schwarz Lemma for Square Roots. Let $F$ be holomorphic in the unit disk. Suppose that $F$ has only multiple zeros and that $|F(z)|<1$ for $|z|<1$. Then $\left|F^{\prime}(0)\right|^{2} \leq 4|F(0)|$.

Note that the conclusion just says that $\left|G^{\prime}(0)\right| \leq 1$ for $G=\sqrt{F}$. Nehari's proof of the Schwarz Lemma for multivalued functions is based on Ahlfors's [6] extension of the Schwarz Lemma. This is also the main tool in Robinson's [29] proof of Theorems N.1 and N.2, so the proof below of these theorems has something in common with Robinson's proof (though there are also significant differences). For completeness we describe the method of Ahlfors and Nehari.

Proof of the Schwarz Lemma for square roots. We may assume $F$ is holomorphic in the closed unit disk and satisfies $|F(z)|<1$ there, because otherwise we can consider $F(r z)$ instead of $F(z)$ for $r<1$, and then take the limit as $r \rightarrow 1$. 
We put

$$
u(z)=\log \frac{\left|F^{\prime}(z)\right|}{2 \sqrt{|F(z)|}(1-|F(z)|)} \quad \text { and } \quad v(z)=\log \frac{1}{1-|z|^{2}} .
$$

Then $u(z) \rightarrow-\infty$ if $z$ tends to a zero of $F$ of multiplicity at least three, but $u$ is finite and $C^{\infty}$ at all other points of the unit disk, including the double zeros of $F$. We also have $v(z) \rightarrow \infty$ as $|z| \rightarrow 1$. Thus the function $w:=u-v$ attains its maximum at some point $z_{0}$ in the unit disk where it is $C^{\infty}$. This implies that $\Delta w\left(z_{0}\right) \leq 0$. Now

$$
\Delta u=4 e^{2 u} \text { and } \Delta v=4 e^{2 v}
$$

so that

$$
\Delta w\left(z_{0}\right)=4\left(e^{2 u\left(z_{0}\right)}-e^{2 v\left(z_{0}\right)}\right)
$$

Thus

$$
4\left(e^{2 u\left(z_{0}\right)}-e^{2 v\left(z_{0}\right)}\right) \leq 0,
$$

and hence $u\left(z_{0}\right) \leq v\left(z_{0}\right)$. From the definition of $z_{0}$ we thus deduce that $u(z)-v(z)=$ $w(z) \leq w\left(z_{0}\right)=u\left(z_{0}\right)-v\left(z_{0}\right) \leq 0$ for $|z|<1$. This yields

$$
\frac{\left|F^{\prime}(z)\right|}{2 \sqrt{|F(z)|(1-|F(z)|)}} \leq \frac{1}{1-|z|^{2}} .
$$

The conclusion follows from the special case $z=0$.

Proof of Theorems N.1 and N.2. We have already seen in $\S 1$ that each of the two results can easily be deduced from the other one. Assume now that Theorem N. 1 is false, so that there exist a domain $D$ and $a_{j} \in \hat{\mathbb{C}}, j=1, \ldots, 5$, such that $\mathcal{F}_{N}\left(D,\left\{a_{j}\right\}_{j=1}^{5}\right)$ is not normal. An application of the rescaling lemma shows that $\mathcal{F}_{N}\left(\mathbb{C},\left\{a_{j}\right\}_{j=1}^{5}\right)$ contains a non-constant function $f$ whose spherical derivative is bounded. We may assume that $a_{j} \neq \infty$ for all $j \in\{1, \ldots, 5\}$. Consider the entire function

$$
g(z)=\frac{f^{\prime}(z)^{2}}{\prod_{j=1}^{5}\left(f(z)-a_{j}\right)}
$$

clearly, $g \not \equiv 0$. Since $f$ has bounded spherical derivative, $g$ is small when $f$ is large. Thus $g$ is non-constant, and there exists a sequence $\left(z_{n}\right)$ such that $g\left(z_{n}\right) \rightarrow \infty$; hence $f\left(z_{n}\right) \nrightarrow \infty$. We consider $h_{n}(z)=f\left(z+z_{n}\right)$ and, passing to a subsequence if necessary, may assume that $h_{n}$ converges locally uniformly to some meromorphic function $h: \mathbb{C} \rightarrow \hat{\mathbb{C}}$. It follows that $h(z) \equiv a_{k}$ for some $k \in\{1, \ldots, 5\}$, because otherwise

$$
g\left(z+z_{n}\right) \rightarrow \frac{h^{\prime}(z)^{2}}{\prod_{j=1}^{5}\left(h(z)-a_{j}\right)} \neq \infty
$$


which contradicts $g\left(z_{n}\right) \rightarrow \infty$.

For sufficiently large $n$, the function $F=h_{n}-a_{k}$ satisfies the hypotheses of the Schwarz Lemma for square roots. Thus $\left|f^{\prime}\left(z_{n}\right)\right|^{2}=\left|F^{\prime}(0)\right|^{2} \leq 4|F(0)|=$ $4\left|f\left(z_{n}\right)-a_{k}\right|$ and hence

$$
\left|g\left(z_{n}\right)\right| \leq \frac{4}{\prod_{j \neq k}\left|f\left(z_{n}\right)-a_{j}\right|},
$$

which is a contradiction since $f\left(z_{n}\right) \rightarrow a_{k}$ and $g\left(z_{n}\right) \rightarrow \infty$.

Remark. We note that if all $a_{k}$-points of $f$ have even multiplicity, and if $F$ is as in the above proof, then $\sqrt{F}$ can be defined as a single-valued function, and thus the classical Schwarz Lemma (applied to this function) suffices. (Alternatively, we observe that $F \rightarrow 0$ as $n \rightarrow \infty$ so that $\sqrt{F} \rightarrow 0$ and hence $F^{\prime} / 2 \sqrt{F} \rightarrow 0$, which yields $F^{\prime}(0)^{2} / F(0) \rightarrow 0$.) Hence we obtain a fairly easy proof of the result that $a$ non-constant meromorphic function in the plane cannot take five values with only even multiplicities, as well as of the corresponding normality criterion.

\section{A proof of the rescaling lemma}

By $f^{\#}$ we denote the spherical derivative of a meromorphic function $f$. Recall that Marty's normality criterion says that a family $\mathcal{F}$ of meromorphic functions is normal if and only if the family $\left\{f^{\#}\right\}_{f \in \mathcal{F}}$ is locally bounded; see, e. g., [8, p. 218], $[22$, p. 158$]$ or $[30$, p. 75$]$.

To prove the rescaling lemma, suppose that $\mathcal{F}$ is not normal. By Marty's criterion, there exists a sequence $\left(\zeta_{k}\right)$ in $D$ tending to a point $\zeta_{0} \in D$ and a sequence $\left(f_{k}\right)$ in $\mathcal{F}$ such that $f_{k}^{\#}\left(\zeta_{k}\right) \rightarrow \infty$. Without loss of generality, we may assume that $\zeta_{0}=0$ and that $\{z:|z| \leq 1\} \subset D$. Choose $z_{k}$ satisfying $\left|z_{k}\right| \leq 1$ such that $M_{k}:=f_{k}^{\#}\left(z_{k}\right)\left(1-\left|z_{k}\right|\right)=\max _{|z| \leq 1} f_{k}^{\#}(z)(1-|z|)$. Then $M_{k} \geq f_{k}^{\#}\left(\zeta_{k}\right)\left(1-\left|\zeta_{k}\right|\right)$ and hence $M_{k} \rightarrow \infty$. Define $\rho_{k}=1 / f_{k}^{\#}\left(z_{k}\right)$. Then $\rho_{k} \leq 1 / M_{k}$ so that $\rho_{k} \rightarrow 0$. Since $\left|z_{k}+\rho_{k} z\right|<1$ for $|z|<\left(1-\left|z_{k}\right|\right) / \rho_{k}=M_{k}$, the function $g_{k}(z)=f_{k}\left(z_{k}+\rho_{k} z\right)$ is defined for $|z|<M_{k}$ and satisfies

$$
g_{k}^{\#}(z)=\rho_{k} f_{k}^{\#}\left(z_{k}+\rho_{k} z\right) \leq \frac{1-\left|z_{k}\right|}{1-\left|z_{k}+\rho_{k} z\right|} \leq \frac{1-\left|z_{k}\right|}{1-\left|z_{k}\right|-\rho_{k}|z|}=\frac{1}{1-|z| / M_{k}}
$$

there. By Marty's criterion, the sequence $\left(g_{k}\right)$ is normal in $\mathbb{C}$ and thus has a subsequence which converges locally uniformly in $\mathbb{C}$. Without loss of generality, we may assume that $g_{k} \rightarrow f$ for some $f: \mathbb{C} \rightarrow \hat{\mathbb{C}}$ and $z_{k} \rightarrow z_{0}$ for some $z_{0} \in D$. Since $g_{k}^{\#}(0)=1$ for all $k$, we have $f^{\#}(0)=1$, so that $f$ is non-constant. Clearly, we also have $f^{\#}(z) \leq 1$ for all $z \in \mathbb{C}$. 


\section{Further remarks}

1 Theorems A.1, A.2, N.1 and N.2 are actually special cases of more general results. Here we only formulate a generalization of Theorem N.2.

Theorem. Let $f: \mathbb{C} \rightarrow \hat{\mathbb{C}}$ be a non-constant meromorphic function, $q \in \mathbb{N}$, and $a_{1}, \ldots, a_{q} \in \hat{\mathbb{C}}$ distinct. Suppose that all $a_{j}$-points of $f$ have multiplicity at least $m_{j}$. Then

$$
\sum_{j=1}^{q}\left(1-\frac{1}{m_{j}}\right) \leq 2 .
$$

If $f$ does not take a value $a_{j}$, then we can take $m_{j}=\infty$ and $1-1 / m_{j}=1$. So this theorem contains Picard's theorem, and it also follows that an entire function has at most two finite perfectly branched values.

The above theorem may also be proved using the methods of $\S 3$. (Here we define $g(z)=f^{\prime}(z)^{M} / \prod_{j=1}^{q}\left(f(z)-a_{j}\right)^{\left(m_{j}-1\right) M / m_{j}}$, where $M$ is the least common multiple of the $m_{j}$, and later use a Schwarz lemma for $m$-th roots giving $\left|F^{\prime}(0)\right|^{\text {rn }} \leq$ $m^{m}|F(0)|^{m-1}$ if $F$ has only zeros of multiplicity at least $m$. If $m_{j}=\infty$ for some $j$, we replace $m_{j}$ by a large integer.) Again, the proof is particularly easy in the special case where the multiplicity of each $a_{j}$-point is a multiple of $m_{j}$. For $q=3$ this special case of the theorem goes back to Carathéodory [18].

We leave it to the reader to state (or look up in the references given in $\S 1$ ) the analogous generalizations of Theorems A.1, A.2 and N.1 and to check that they can be deduced from the result given above with the rescaling lemma and the methods of $\S 2$.

2 As already mentioned, the generalization of Theorem N.2 given above contains Picard's theorem (and thus the corresponding generalization of Theorem N.1 contains Montel's theorem). I consider the method used as a serious candidate in the competition for the simplest proof of the theorems of Picard and Montel (although I admit that the proof in [37, p. 218] is hard to beat). For completeness, we summarize the argument.

Suppose that Montel's (or Picard's) theorem does not hold. An application of the rescaling lemma shows that there then exists a non-constant function $f$ which is meromorphic in the plane, omits three values $a_{1}, a_{2}, a_{3} \in \mathbb{C}$ and has bounded spherical derivative. The function $g(z)=f^{\prime}(z)^{4} / \prod_{j=1}^{3}\left(f(z)-a_{j}\right)^{3}$ is then entire and close to zero where $f(z)$ is large. Since $g(z) \not \equiv 0$, there exists a sequence $\left(z_{n}\right)$ such that $g\left(z_{n}\right) \rightarrow \infty$; hence $f\left(z_{n}\right) \nrightarrow \infty$. By normality, we can assume that $f\left(z+z_{n}\right) \rightarrow h(z)$ locally uniformly in $\mathbb{C}$ for some meromorphic 
function $h$. We deduce that $h(z) \equiv a_{k}$ for some $k \in\{1,2,3\}$, since otherwise $g\left(z+z_{n}\right) \rightarrow h^{\prime}(z)^{4} / \prod_{j=1}^{3}\left(h(z)-a_{j}\right)^{3} \neq \infty$, contradicting $g\left(z_{n}\right) \rightarrow \infty$. It follows that $\left(f\left(z+z_{n}\right)-a_{k}\right)^{1 / 4} \rightarrow 0$. This implies that

$$
\frac{f^{\prime}\left(z+z_{n}\right)}{4\left(f\left(z+z_{n}\right)-a_{k}\right)^{3 / 4}} \rightarrow 0
$$

and thus $f^{\prime}\left(z_{n}\right)^{4} /\left(f\left(z_{n}\right)-a_{k}\right)^{3} \rightarrow 0$, contradicting $g\left(z_{n}\right) \rightarrow \infty$.

3 The deduction of Theorem X.1 from Theorem X.2 and vice versa is an example of Bloch's Principle, which says that the family of all functions meromorphic in some domain which have a certain property there is likely to be normal if there is no non-constant meromorphic function in the plane which has this property. Zalcman introduced the rescaling lemma in order to make this heuristic principle rigorous and to prove it for certain properties.

The paper [14] to which this principle is usually traced actually contains two principles. Bloch states the first of these as "Nihil est in infinito quod non prius fuerit in finito," and it is this that seems to have been interpreted later as explained above. (I could not, however, find this explicit formulation in Bloch's paper.) Bloch calls the second principle "le principe de continuité topologique." To illustrate it, he quotes the result that the family of holomorphic functions having three fixed finite values as perfectly branched values is normal and contrasts it with the result that the family of all holomorphic functions having no simple island over three given disks with pairwise disjoint closures is normal. (Bloch notes that "three" has to be replaced by "five" for meromorphic functions.) The arguments in $\S 2$ can be considered as a confirmation of Bloch's belief that the results mentioned should be equivalent in the sense that either one can easily be deduced from the other one.

So Bloch actually "knew" the five islands theorem in the special case that the domains $D_{j}$ are disks, although his arguments for it did not constitute a rigorous proof. We note that, in the early versions of his theory, Ahlfors [1, 2, 4] also considered only the case where the $D_{j}$ are disks. Ahlfors gives an interesting discussion of Bloch's second principle in [3, pp. 202-203].

For a thorough discussion of Bloch's first principle, see the book by Schiff [30, Chapter 4] or the survey by Zalcman [37]. The latter paper also contains various other applications of the rescaling lemma. Recently, Eremenko [21] has given a simple proof of the celebrated covering theorem of Bloch (and, in fact, a generalization of this result to quasiregular mappings in higher dimensions) using the rescaling lemma. Here a shrinking of disks to points similar to that in $\S 2$ is used. 
The fact that the function $f$ occurring in the rescaling lemma has bounded spherical derivative is not used in $\S 2$, but it is essential in $\S 3$. This fact has proved useful in other instances as well: instead of considering arbitrary meromorphic functions, it suffices for certain problems to consider functions with bounded spherical derivative. For a discussion of this aspect of the rescaling lemma, see $[37$, p. 226f]. This argument has also been used in [17].

4 Quasiconformal mappings, and in particular the existence theorem mentioned in $\S 2$, have become an indispensable tool in the modern treatment of complex dynamics (see, e.g., $[12,19,33]$ ). The Ahlfors five islands theorem has also found applications in complex dynamics. The first such application is due to Baker [9], who used Theorem A.1 to prove that repelling periodic points are dense in the Julia set of an entire function. Later, Schwick [31] gave a different and more elementary proof of this fact, using Theorem N.2 and the rescaling lemma instead. (It was this paper of Schwick which led me to ask whether one could also obtain Theorem A. 2 from Theorem N.2 and the rescaling lemma. Meanwhile, however, there is an even more elementary proof of Baker's result by Bargmann [11], where only the rescaling lemma and a few simple facts from complex dynamics are needed.) Schwick's proof has been extended by Bolsch [16] to meromorphic functions with countably many essential singularities. For transcendental meromorphic functions in the plane, the density of repelling periodic points in the Julia set had been proved earlier by Baker, Kotus and Lü [10], using a version of the five islands theorem.

There are further applications of Theorem A.1 in complex dynamics, e.g., it has been used to estimate the Hausdorff dimension of Julia sets [32], to study connectedness properties of Julia sets [20] and to prove the existence of periodic points of given periods [13].

The proofs of Theorems A.1 and A.2 given here may thus be of particular interest for people working in complex dynamics. They have the existence theorem for quasiconformal mappings in their toolbox anyway, and everything else that is needed is fairly simple and elementary.

\section{REFERENCES}

[1] L. V. Ahlfors, Sur une généralisation du théorème de Picard, C. R. Acad. Sci. Paris 194 (1932), 245-247, and Collected Papers, Vol. I, Birkhäuser, Boston, Basel, Stuttgart, 1982, pp. 145-147.

[2] L. V. Ahlfors, Sur les fonctions inverses des fonctions méromorphes, C. R. Acad. Sci. Paris 194 (1932), 1145-1147, and Collected Papers, Vol. 1, pp. 149-151.

[3] L. V. Ahlfors, Quelques propriétés des surfaces de Riemann correspondant aux fonctions méromorphes, Bull. Soc. Math. France 60 (1932), 197-207, and Collected Papers, Vol. I, pp. 152-162. 
[4] L. V. Ahlfors, Über die Kreise die von einer Riemannschen Fläche schlicht überdeckt werden, Comment. Math. Helv. 5 (1933), 28-38, and Collected Papers, Vol. I, pp. 163-173.

[5] L. V. Ahlfors, Zur Theorie der Überlagerungsfächen, Acta Math. 65 (1935), 157-194, and Collected Papers, Vol. I, pp. 214-251.

[6] L. V. Ahlfors, An extension of Schwarz's lemma, Trans. Amer. Math. Soc. 43 (1938), 359-364, and Collected Papers, Vol. I, pp. 350-355.

[7] L. V. Ahlfors, Lectures on Quasiconformal Mappings, Van Nostrand, Princeton, 1966.

[8] L. V. Ahlfors, Complex Analysis, 2nd ed., McGraw-Hill, New York, 1966.

[9] I. N. Baker, Repulsive fuxpoints of entire functions, Math. Z. 104 (1968), 252-256.

[10] I. N. Baker, J. Kotus and Y. Lü, Iterates of meromorphic functions, I, Ergodic Theory Dynam. Systems 11 (1991), 241-248.

[11] D. Bargmann, Simple proofs of some fundamental properties of the Julia set, Ergodic Theory Dynam. Systems, to appear.

[12] A. F. Beardon, Iteration of Rational Functions, Springer, New York, Berlin, Heidelberg, 1991.

[13] W. Bergweiler, Periodic points of entire functions: proof of a conjecture of Baker, Complex Variables Theory Appl. 17 (1991), 57-72.

[14] A. Bloch, La conception actuelle de la théorie des fonctions entières et méromorphes, Enseign. Math. 25 (1926), 83-103.

[15] A. Bloch, Les fonctions holomorphes et méromorphes dans le cercle-unité, Gauthiers-Villars, Paris, 1926.

[16] A. Bolsch, Repulsive periodic points of meromorphic functions, Complex Variables Theory Appl. 31 (1996), 75-79.

[17] M. Bonk and A. Eremenko, Schlicht regions for entire and meromorphic functions, preprint, 1998.

[18] C. Carathéodory, Sur quelques généralisations du théorème de M. Picard, C. R. Acad. Sci. Paris 141 (1905), 1213-1215.

[19] L. Carleson and T. W. Gamelin, Complex Dynamics, Springer, New York, Berlin, Heidelberg, 1993.

[20] P. Domínguez, Connectedness properties of Julia sets of transcendental entire functions, Complex Variables Theory Appl. 32 (1997), 199-215.

[21] A. Eremenko, Bloch radius, normal families and quasiregular mappings, Proc. Amer. Math. Soc., to appear.

[22] W. K. Hayman, Meromorphic Functions, Clarendon Press, Oxford, 1964.

[23] O. Lehto, Quasiconformal homeomorphisms and Beltrami equations, in Discrete Groups and Automorphic Forms (W. J. Harvey, ed.), Academic Press, London, New York, San Francisco, 1977, pp. 121-142.

[24] O. Lehto and K. I. Virtanen, Quasiconformal Mappings in the Plane, Springer, Berlin, Heidelberg, New York, 1973.

[25] A. J. Lohwater and Ch. Pommerenke, On normal meromorphic functions, Ann. Acad. Sci. Fenn., Ser. A., no. 550 (1973).

[26] Z. Nehari, A generalization of Schwarz' lemma, Duke Math. J. 14 (1947), 1035-1049.

[27] R. Nevanlinna, Le théorème de Picard-Borel et la théorie des fonctions méromorphes, GauthiersVillars, Paris, 1929.

[28] R. Nevanlinna, Eindeutige analytische Funktionen, Springer, Berlin, Göttingen, Heidelberg, 1953.

[29] R. M. Robinson, A generalization of Picard's and related theorems, Duke Math. J. 5 (1939), 118-132.

[30] J. Schiff, Normal Families, Springer, New York, Berlin, Heidelberg, 1993. 
[31] W. Schwick, Repelling periodic points in the Julia set, Bull. London Math. Soc. 29 (1997), 314-316.

[32] G. M. Stallard, The Hausdorff dimension of Julia sets of meromorphic functions, J. London Math. Soc. (2) 49 (1994), 281-295.

[33] N. Steinmetz, Rational Iteration, Walter de Gruyter, Berlin, 1993.

[34] M. Tsuji, Potential Theory in Modern Function Theory, Maruzen, Tokyo, 1959.

[35] G. Valiron, Familles normales et quasi-normales de fonctions méromorphes, Gauthiers-Villars, Paris, 1929.

[36] L. Zalcman, A heuristic principle in complex function theory, Amer. Math. Monthly 82 (1975), 813-817.

[37] L. Zalcman, Normal families: new perspectives, Bull. Amer. Math. Soc. 35 (1998), 215-230.

Walter Bergweiler

MATHEMATISCHES SEMINAR

CHRISTIAN-ALBRECHTS-UNIVERSITÄT ZU KIEL

LUDEWIG-MEYN-STR. 4

D-24098 KIEL, GERMANY

email: bergweiler@math.uni-kiel.de

(Received September 16, 1998) 